\title{
On the critical exponent in an isoperimetric inequality for chords
}

\author{
Pavel Exner ${ }^{1,3}$, Martin Fraas ${ }^{2,3}$, Evans M. Harrell II ${ }^{4}$ \\ 1) Nuclear Physics Institute, Czech Academy of Sciences, 25068 Řež near Prague, \\ 2) Faculty of Mathematics and Physics, Charles University, V Holešovičkách 2, \\ 18040 Prague \\ 3) Doppler Institute, Czech Technical University, Břehová 7, 11519 Prague, \\ Czechia \\ 4) School of Mathematics, Georgia Institute of Technology, Atlanta, GA 30332, \\ U.S.A. \\ e-mail: exner@ujf.cas.cz,fraas@ujf.cas.cz,harrell@math.gatech.edu
}

The problem of maximizing the $L^{p}$ norms of chords connecting points on a closed curve separated by arclength $u$ arises in electrostatic and quantum-mechanical problems. It is known that among all closed curves of fixed length, the unique maximizing shape is the circle for $1 \leq p \leq 2$, but this is not the case for sufficiently large values of $p$. Here we determine the critical value $p_{c}(u)$ of $p$ above which the circle is not a local maximizer finding, in particular, that $p_{c}\left(\frac{1}{2} L\right)=\frac{5}{2}$. This corrects a claim made in [3].

If $\Gamma(s)$ describes a planar curve of parametrized by arclength $s$ and $L$ is its total length, then

$$
\left(\frac{1}{L} \int_{0}^{L}|\Gamma(s+u)-\Gamma(s)|^{p} \mathrm{~d} s\right)
$$

describes the $L^{p}$-mean of the Euclidean length of the chords connecting points separated by arclength $u$. A reasonable geometric question is to determine the shape that maximizes this quantity for any given value of $p$. Some phys- 
ical phenomena have recently been shown to have connections to this geometric question:

1. What shape will a loop in $\mathbb{R}^{3}$ carrying a uniform electric charge assume at equilibrium? That is, what is the minimum of the potential energy due to Coulomb repulsion? For this problem see $[1,4]$ and references therein.

2. What is the shape of a loop $\Gamma$ of length $L$ that maximizes the groundstate energy of a leaky quantum graph in the plane? That is, how can the fundamental eigenvalue of the leaky-graph Hamiltonian $-\Delta-$ $\alpha \delta(x-\Gamma)$ acting in $L^{2}\left(\mathbb{R}^{2}\right)$ be maximized? This problem was considered in $[2,3]$ and references therein.

In both of these problems it turns out that the solution reduces to considering the $L^{p}$-means of chords, specifically to establishing the validity of

$$
C_{L}^{p}(u): \quad c_{\Gamma}^{p}(u):=\int_{0}^{L}|\Gamma(s+u)-\Gamma(s)|^{p} \mathrm{~d} s \leq \frac{L^{1+p}}{\pi^{p}} \sin ^{p} \frac{\pi u}{L},
$$

with $p=1$ and $u \in\left(0, \frac{1}{2} L\right]$. In other words, can it be shown that the global maximizer is a planar circle of radius $\frac{1}{2 \pi} L$, which by an elementary calculation attains the value on the right side? By a convexity argument it suffices to prove the inequality for any larger value of $p$ to establish it for smaller values.

The inequality $C_{L}^{p}(u)$ was established for the first time over forty years ago by Lükö [5] for $p=2$. The same claim was demonstrated more recently in different ways in $[1,3]$; see also a local proof in [2]. It is natural to consider the maximal value of $p$ for which the inequality holds. The best upper estimate so far, $p \approx 3.15$, was obtained in [3] by investigating a stadium-shaped $\Gamma$.

Our aim here is to improve this result. Using the method of [2] we shall show that among all planar closed curves, $c_{\Gamma}^{p}(u)$ is locally maximized by a circle if $p<\frac{5}{2}$, and to find a local critical value of $p$ for "shorter" chords. Since the inequality in question has obvious scaling properties, it is sufficient to consider the case $L=2 \pi$. We keep a general $L$ in the main claims for the convenience of the reader, but otherwise we will work with the particular value $L=2 \pi$.

Without loss of generality we may assume that the $\Gamma$ is a $\mathcal{C}^{2}$-smooth curve, the validity of the result being extended to less regular loops by continuity. 
Using the notation of $[2$, Sec. 5$]$ the quantity $c_{\Gamma}^{p}(u)$ can be cast into the form

$$
c_{\Gamma}^{p}(u)=\int_{0}^{L} \mathrm{~d} s\left[\int_{s}^{s+u} \mathrm{~d} s^{\prime} \int_{s}^{s+u} \mathrm{~d} s^{\prime \prime} \cos \left(\int_{s^{\prime}}^{s^{\prime \prime}} \gamma(\tau) \mathrm{d} \tau\right)\right]^{p / 2},
$$

where $\gamma:=\dot{\Gamma}_{2} \ddot{\Gamma}_{1}-\dot{\Gamma}_{1} \ddot{\Gamma}_{2}$ is the signed curvature of $\Gamma$. Recall that the knowledge of $\gamma$ allows us reconstruct $\Gamma$ up to Euclidean transformations by

$$
\Gamma(s)=\left(\int_{0}^{s} \cos \beta(t) \mathrm{d} t, \int_{0}^{s} \sin \beta(t) \mathrm{d} t\right),
$$

where $\beta(s):=\int_{0}^{s} \gamma(t) \mathrm{d} t$ is the angle between the tangent vectors at $t=s$ and the initial point, $t=0$. We shall refer to this as the bending of the arc.

Our aim is to compute the first and second Gâteaux derivatives of the map $\Gamma \mapsto c_{\Gamma}^{p}(u)$ at the circle, $\Gamma=C$, and to demonstrate the claim by looking into their properties. Consequently, we shall consider gentle deformations of a circle, which can be characterized by variations of the curvature

$$
\gamma(s)=\frac{2 \pi}{L}+\varepsilon g(s)
$$

where $g$ is a continuous $L$-periodic function and $\varepsilon$ is small in the sense that $\varepsilon\|g\|_{\infty} \ll 1$. The periodicity and continuity make it possible to express $g$ through its Fourier series

$$
g(s)=a_{0}+\sum_{n=1}^{\infty} a_{n} \sin \left(\frac{2 \pi n s}{L}\right)+b_{n} \cos \left(\frac{2 \pi n s}{L}\right)
$$

with $\{a\},\{b\} \in \ell^{2}$. We are interested in closed curves $\Gamma$, so we ask now how this property is reflected in Fourier series.

Proposition 1 The tangent to $\Gamma \in \mathcal{C}^{2}$ corresponding to (2) is periodic with period $L$ if and only if $a_{0}=0$. Furthermore, $\Gamma(0)=\Gamma(L)+\mathcal{O}\left(\varepsilon^{3}\right)$ provided that

$$
a_{1}=b_{1}=0 \quad \text { and } \quad \sum_{n=2}^{\infty} \frac{b_{n} b_{n+1}+a_{n} a_{n+1}}{n(n+1)}=\sum_{n=2}^{\infty} \frac{a_{n+1} b_{n}-b_{n+1} a_{n}}{n(n+1)}=0 .
$$


Proof: As mentioned above, we may henceforth set $L=2 \pi$. In view of the definition of $\beta(s)$ it is clear that the tangent vector is continuous if $\beta(L)=2 \pi$. In our case the bending function is

$$
\beta(s)=s+\varepsilon \int_{0}^{s} g(t) \mathrm{d} t=: s+\varepsilon b(s),
$$

and the condition simplifies to $\int_{0}^{2 \pi} g(t) \mathrm{d} t=0$ which holds iff $a_{0}=0$. In view of (1) the fact that $\Gamma$ is closed means

$$
\left(\int_{0}^{2 \pi} \cos \beta(s) \mathrm{d} s, \int_{0}^{2 \pi} \sin \beta(s) \mathrm{d} s\right)=(0,0) .
$$

For the terms on the right side of the last equation we have the expansion

$$
\begin{aligned}
& \cos \beta(s)=\left(1-\frac{1}{2} \varepsilon^{2} b^{2}(s)\right) \cos s-\varepsilon b(s) \sin s+\mathcal{O}\left(\varepsilon^{3}\right) \\
& \sin \beta(s)=\left(1-\frac{1}{2} \varepsilon^{2} b^{2}(s)\right) \sin s+\varepsilon b(s) \cos s+\mathcal{O}\left(\varepsilon^{3}\right)
\end{aligned}
$$

Up to the third order in $\varepsilon$ we get thus the conditions

$$
\begin{aligned}
\int_{0}^{2 \pi} b(s) \cos s \mathrm{~d} s & =\int_{0}^{2 \pi} b(s) \sin s \mathrm{~d} s=0 \\
\int_{0}^{2 \pi} b(s)^{2} \cos s \mathrm{~d} s & =\int_{0}^{2 \pi} b(s)^{2} \sin s \mathrm{~d} s=0 .
\end{aligned}
$$

It is convenient to rewrite the Fourier series for the curvature deformation in the complex form, $g(s)=\sum_{n \neq 0} c_{n} e^{i n s}$ where $c_{-n}=\bar{c}_{n}$ and for $n>0$ we have $c_{n}=\frac{1}{2}\left(b_{n}-i a_{n}\right)$. For $b(s)$ this yields the following series:

$$
b(s)=\sum_{n \neq 0} \frac{i c_{n}}{n}\left[1-e^{i n s}\right] .
$$

Using orthonormality of the trigonometric basis we see that the condition (3) requires $a_{1}=b_{1}=0$. On the other hand, the remaining condition (4) means that the integral $\int_{0}^{L} b(s)^{2} e^{i n s} \mathrm{~d} s$ must vanish; with the help of the above series we can express it in the following way,

$$
-\sum_{n, m \neq 0, \pm 1} \frac{c_{n} c_{m}}{n m} \int_{0}^{2 \pi} e^{i s}\left[1-e^{i n s}\right]\left[1-e^{i m s}\right] \mathrm{d} s=\sum_{n \neq 0, \pm 1} \frac{c_{n} \bar{c}_{n+1}}{n(n+1)}
$$


and taking the real and imaginary part we arrive at the claimed identities for $\left\{a_{n}\right\}$ and $\left\{b_{n}\right\}$.

After this preliminary let us turn to our proper subject. The Gâteaux derivative of the functional (1) in the direction $g$ is

$$
\begin{aligned}
\mathrm{D}_{g} c_{\Gamma}^{p}(u) & =\left.\frac{\partial c_{\Gamma}^{p}(u)}{\partial \varepsilon}\right|_{\varepsilon=0} \\
= & -\frac{p}{2}\left[4 \sin ^{2} \frac{u}{2}\right]^{p / 2-1} \int_{0}^{2 \pi} \mathrm{d} s \int_{s}^{s+u} \mathrm{~d} s^{\prime} \int_{s}^{s+u} \mathrm{~d} s^{\prime \prime} \sin \left(\int_{s^{\prime}}^{s^{\prime \prime}} \mathrm{d} t\right) \int_{s^{\prime}}^{s^{\prime \prime}} g(\tau) \mathrm{d} \tau
\end{aligned}
$$

again for $L=2 \pi$, and the second derivative is

$$
\begin{aligned}
& \mathrm{D}_{g}^{2} c_{\Gamma}^{p}(u)=\left.\frac{\partial^{2} c_{\Gamma}^{p}(u)}{\partial \varepsilon^{2}}\right|_{\varepsilon=0} \\
& =\frac{p}{2}\left(\frac{p}{2}-1\right)\left[4 \sin ^{2} \frac{u}{2}\right]^{p / 2-2} \int_{0}^{2 \pi} \mathrm{d} s\left(\int_{s}^{s+u} \mathrm{~d} s^{\prime} \int_{s}^{s+u} \mathrm{~d} s^{\prime \prime} \sin \left(s^{\prime \prime}-s^{\prime}\right) \int_{s^{\prime}}^{s^{\prime \prime}} g(\tau) \mathrm{d} \tau\right)^{2} \\
& \quad-\frac{p}{2}\left[4 \sin ^{2} \frac{u}{2}\right]^{p / 2-1} \int_{0}^{2 \pi} \mathrm{d} s \int_{s}^{s+u} \mathrm{~d} s^{\prime} \int_{s}^{s+u} \mathrm{~d} s^{\prime \prime} \cos \left(s^{\prime \prime}-s^{\prime}\right)\left(\int_{s^{\prime}}^{s^{\prime \prime}} g(\tau) \mathrm{d} \tau\right)^{2} .
\end{aligned}
$$

Rearranging the integrals in (5) we get

$$
\begin{aligned}
& \int_{0}^{2 \pi} \mathrm{d} s \int_{s}^{s+u} \mathrm{~d} s^{\prime} \int_{s}^{s+u} \mathrm{~d} s^{\prime \prime} \sin \left(s^{\prime \prime}-s^{\prime}\right) \int_{s^{\prime}}^{s^{\prime \prime}} g(\tau) \mathrm{d} \tau \\
&=\int_{0}^{2 \pi} \mathrm{d} \tau \int_{\tau-u}^{\tau} \mathrm{d} s \int_{s}^{\tau} \mathrm{d} s^{\prime} \int_{\tau}^{s+u} \mathrm{~d} s^{\prime \prime} \sin \left(s^{\prime \prime}-s^{\prime}\right) g(\tau) \mathrm{d} \tau \\
&=\left(4 \sin ^{2} u+u \sin u\right) \int_{0}^{L} g(\tau) \mathrm{d} \tau=0,
\end{aligned}
$$

which shows that for every $p>0$ the circle is either an extremal or a saddle point. (There are no solutions to $4 \sin u=-u$ in $[-\pi, \pi]$.) In the next step 
we analyze the second derivative to distinguish in between these two cases. Not surprisingly, the answer depends on the value of $u$. Our main result reads

Theorem 2 For a fixed arc length $u \in\left(0, \frac{1}{2} L\right]$ define

$$
p_{c}(u):=\frac{4-\cos \left(\frac{2 \pi u}{L}\right)}{1-\cos \left(\frac{2 \pi u}{L}\right)},
$$

then we have the following alternative. For $p>p_{c}(u)$ the circle is either a saddle point or a local minimum, while for $p<p_{c}(u)$ it is a local maximum of the map $\Gamma \mapsto c_{\Gamma}^{p}(u)$.

Before passing to the proof let us make a pair of comments.

\section{Remarks 3}

1. It will be seen from the proof that in the critical case $p=p_{c}(u)$, the higher order derivatives of $c_{\Gamma}^{p}(u)$ come into play. We shall not address the critical case here.

2. It is natural to expect and easy to verify that for $p>p_{c}$ circle is in fact a saddle point of the functional.

Proof: We put again $L=2 \pi$ and analyze the terms of the second derivative (6) separately. By a straightforward computation using orthonormality of the trigonometric basis the iterated integral in the first term equals

$$
\sum_{n=2}^{\infty}\left[a_{n}^{2} \mathrm{fs}_{1}(n, u, p)+b_{n}^{2} \mathrm{fc}_{1}(n, u, p)\right]
$$

where

$$
\mathrm{fs}_{1}(n, u, p)=\mathrm{fc}_{1}(n, u, p):=\frac{16 \pi}{n-n^{3}}\left(-2 n \cos \frac{n u}{2} \sin ^{2} \frac{u}{2}+\sin u \sin \frac{n u}{2}\right)^{2} .
$$


In the second term we rearrange the integrals before using the Fourier series,

$$
\begin{aligned}
& \int_{s}^{s+u} \mathrm{~d} s^{\prime} \int_{s}^{s+u} \mathrm{~d} s^{\prime \prime} \int_{s^{\prime}}^{s^{\prime \prime}} \mathrm{d} \tau \int_{s^{\prime}}^{s^{\prime \prime}} \mathrm{d} \tau^{\prime} \cos \left(s^{\prime \prime}-s^{\prime}\right) g(\tau) g\left(\tau^{\prime}\right) \\
& =2 \int_{s}^{s+u} \mathrm{~d} \tau \int_{\tau}^{s+u} \mathrm{~d} \tau^{\prime} \int_{s}^{\tau} \mathrm{d} s^{\prime} \int_{\tau^{\prime}}^{s+u} \mathrm{~d} s^{\prime \prime} \cos \left(s^{\prime \prime}-s^{\prime}\right) g(\tau) g\left(\tau^{\prime}\right) \\
& =: \int_{s}^{s+u} \mathrm{~d} \tau \int_{\tau}^{s+u} \mathrm{~d} \tau^{\prime} g(\tau) g\left(\tau^{\prime}\right) \operatorname{lnt}\left(s, \tau, \tau^{\prime}\right) .
\end{aligned}
$$

Hence the full integral in the second term of (6) equals

$$
\begin{aligned}
& \int_{0}^{2 \pi} \mathrm{d} s \int_{s}^{s+u} \mathrm{~d} \tau \int_{\tau}^{s+u} \mathrm{~d} \tau^{\prime} g(\tau) g\left(\tau^{\prime}\right) \operatorname{lnt}\left(s, \tau, \tau^{\prime}\right) \\
= & \int_{0}^{2 \pi} \mathrm{d} \tau \int_{\tau}^{\tau+u} \mathrm{~d} \tau^{\prime} \int_{\tau^{\prime}-u}^{\tau} \mathrm{d} s g(\tau) g\left(\tau^{\prime}\right) \operatorname{lnt}\left(s, \tau, \tau^{\prime}\right)=: \int_{0}^{2 \pi} \mathrm{d} \tau \int_{\tau}^{\tau+u} \mathrm{~d} \tau^{\prime} \operatorname{lnt}_{2}\left(\tau, \tau^{\prime}\right) g(\tau) g\left(\tau^{\prime}\right),
\end{aligned}
$$

where

$$
\operatorname{lnt}_{2}\left(\tau, \tau^{\prime}\right):=2\left(\tau^{\prime}-\tau-u\right)\left(\cos \left(\tau^{\prime}-\tau\right)+\cos u\right)+4\left(-\sin \left(\tau^{\prime}-\tau\right)+\sin u\right)
$$

Finally we use the Fourier series and obtain an expression for the iterated integral in the second term

$$
\int_{0}^{2 \pi} \mathrm{d} s \int_{s}^{s+u} \mathrm{~d} s^{\prime} \int_{s}^{s+u} \mathrm{~d} s^{\prime \prime} \cos \left(s^{\prime \prime}-s^{\prime}\right)\left(\int_{s^{\prime}}^{s^{\prime \prime}} g(\tau) \mathrm{d} \tau\right)^{2}=\sum_{n=2}^{\infty}\left[a_{n}^{2} \mathrm{fs}_{2}(n, u, p)+b_{n}^{2} \mathrm{fc}_{2}(n, u, p)\right]
$$

where

$$
\begin{aligned}
\mathrm{fs}_{2}(n, u, p)=\mathrm{fc}_{2}(n, u, p) & :=\frac{\pi}{n-n^{3}}\left(-6 n^{2}+2 n^{4}-2\left(n^{2}-1\right)^{2} \cos u\right. \\
& \left.+(n+1)^{2} \cos (n-1) u+(n-1)^{2} \cos (n+1) u\right) .
\end{aligned}
$$


Now we put it together and get the second derivative in the form

$$
\mathrm{D}_{g}^{2} c_{\Gamma}^{p}(u)=\sum_{n=2}^{\infty}\left(a_{n}^{2}+b_{n}^{2}\right) \frac{2^{p} \pi \sin ^{p-2}\left(\frac{u}{2}\right)}{8\left(n-n^{3}\right)^{2}} p T(n, u, p),
$$

where

$$
\begin{aligned}
& T(n, u, p):=-\left(2 n^{4}-6 n^{2}-2\left(n^{2}-1\right)^{2} \cos u+(n+1)^{2} \cos (n-1) u\right. \\
& \left.+(n-1)^{2} \cos (n+1) u\right)+2(p-2)\left(-2 n \cos \left(\frac{n u}{2}\right) \sin \left(\frac{u}{2}\right)+2 \cos \left(\frac{u}{2}\right) \sin \left(\frac{n u}{2}\right)\right)^{2} .
\end{aligned}
$$

Since $\sin (u / 2)$ is positive for $u \in(0, \pi)$, the sign of each term in the second derivative series $(8)$ is determined by that of $T(n, u, p)$. The equation

$$
T(2, u, p)=-16(4-p+(p-1) \cos u) \sin ^{4}\left(\frac{u}{2}\right)
$$

gives $T(2, u, p)>0$ for $p>p_{c}(u)$, proving the easier part of the alternative, namely that for $p>p_{c}(u)$ the circle fails to be a local maximum of the map $\Gamma \mapsto c_{\Gamma}^{p}(u)$.

It is easy to check that $T(n, u, p)$ is strictly increasing as a function of $p$. Hence to prove the other part of the theorem it is sufficient to show that $T\left(n, u, p_{c}(u)\right)$ is negative for $n \geq 3$. To this aim we define

$$
S(n, u)=-(1-\cos u) T\left(n, u, p_{c}(u)\right) ;
$$

we next prove that this function is positive for $n \geq 3$.

Inserting the critical exponent $p_{c}(u)$ into (9) we obtain

$$
\begin{aligned}
S(n, u)=-4- & 10 n^{2}+2 n^{4}+2\left(n^{2}-1\right)\left(-2\left(n^{2}-2\right) \cos u+n^{2} \cos ^{2} u\right) \\
+ & 4 \cos (n u)\left(1-n^{2}+\left(2+n^{2}\right) \cos u\right)+12 n \sin u \sin (n u),
\end{aligned}
$$

and using the inequality $(a \sin x+b \cos x)^{2} \leq a^{2}+b^{2}$ we get the bound

$$
\begin{aligned}
S(n, u) \geq-4-10 n^{2}+2 n^{4} & +2\left(n^{2}-1\right)\left(-2\left(n^{2}-2\right) \cos u+n^{2} \cos ^{2} u\right) \\
& -4 \sqrt{\left(1-n^{2}+\left(2+n^{2}\right) \cos u\right)^{2}+9 n^{2} \sin ^{2} u} .
\end{aligned}
$$

Hence $S(n, u)$ is positive whenever

$$
-4-10 n^{2}+2 n^{4}+2\left(n^{2}-1\right)\left(-2\left(n^{2}-2\right) \cos u+n^{2} \cos ^{2} u\right)>0
$$


and

$$
\begin{aligned}
\left(-4-10 n^{2}+2 n^{4}+\right. & \left.2\left(n^{2}-1\right)\left(-2\left(n^{2}-2\right) \cos u+n^{2} \cos ^{2} u\right)\right)^{2} \\
> & 16\left(\left(1-n^{2}+\left(2+n^{2}\right) \cos u\right)^{2}+9 n^{2} \sin ^{2} u\right) .
\end{aligned}
$$

The first condition (10) is a quadratic equation in $\cos u$, and a calculation shows that it is satisfied for $\cos u<1-\frac{6}{n^{2}}$. Using the notation $\cos u=x$, the second condition (11) simplifies to

$$
4 n^{2}\left(n^{2}-1\right)^{2}\left(8+n^{2}(x-1)\right)(x-1)^{3}>0,
$$

which provides us with a slightly stronger condition,

$$
\cos u<1-\frac{8}{n^{2}}
$$

The vicinity of zero has to be regarded separately to prove the positivity of $S(n, u)$ on the interval complementary to (12) . By a straightforward computation the Taylor expansion of $S(n, u)$ around zero equals

$$
S(n, u)=\frac{n^{2} u^{8}}{40}\left(-\frac{1}{9}+\frac{n^{2}}{4}-\frac{n^{4}}{6}+\frac{n^{6}}{36}\right)+\frac{u^{10}}{10 !} R_{10},
$$

where for $n \geq 3$ and $u$ in the complement of $(12)$ the $\mathcal{O}\left(u^{10}\right)$ term is bounded from below by

$$
R_{10} \geq-136 n^{10}
$$

Comparing the reminder with the first term on the right-side of (13), we observe that $S(n, u)$ is positive for

$$
u^{2}<\frac{1}{40}\left(-\frac{1}{9}+\frac{n^{2}}{4}-\frac{n^{4}}{6}+\frac{n^{6}}{36}\right) \frac{10 !}{136 n^{8}} .
$$

Now we use the inequality $\cos u \leq 1-7 / 16 u^{2}$ for $u \in\left(0, \frac{6}{5}\right)$ to compare the intervals (12) and (14). By simple analysis we find out that for $n \geq 4$,

$$
1-\frac{8}{n^{2}} \leq 1-\frac{7}{16} \frac{1}{40}\left(-\frac{1}{9}+\frac{n^{2}}{4}-\frac{n^{4}}{6}+\frac{n^{6}}{36}\right) \frac{10 !}{136 n^{8}}
$$

and hence in this case the union of the intervals covers $(0, \pi)$, which proves that $S(n, u) \geq 0$ holds for $n \geq 4$. 


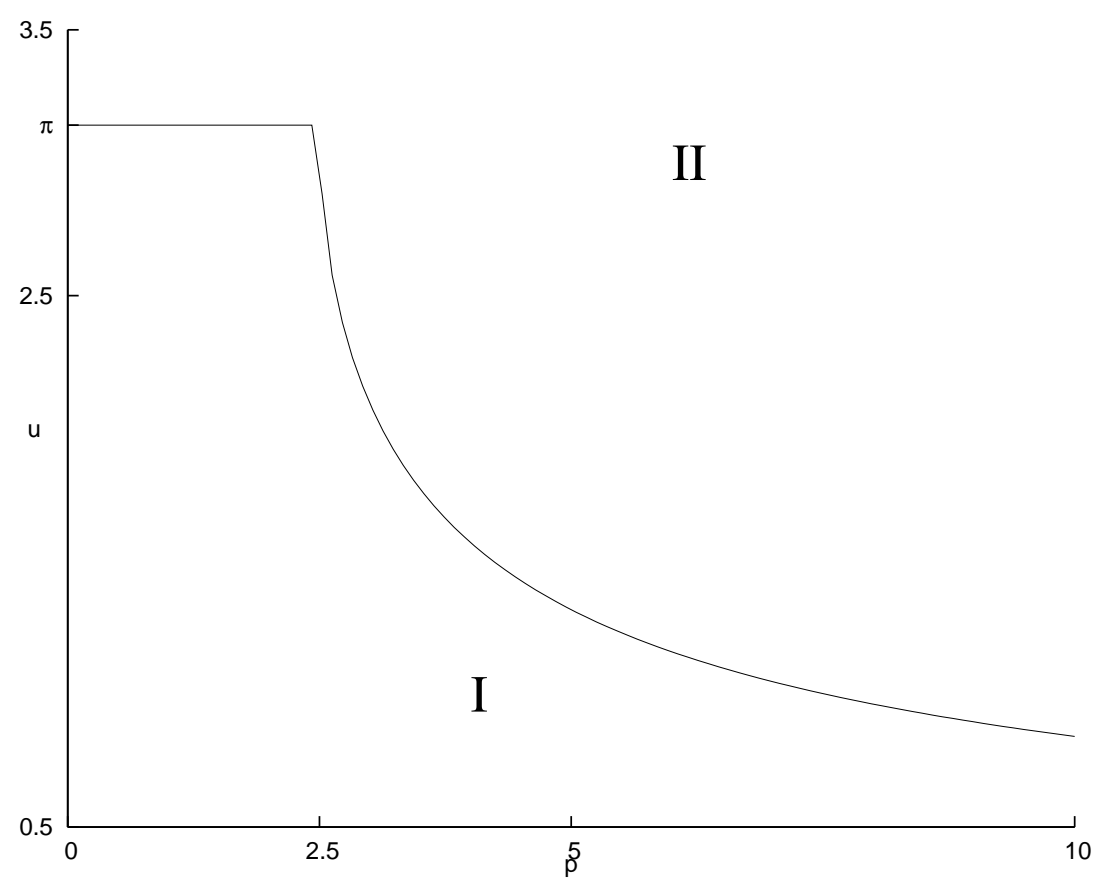

Figure 1: The relation between the critical exponent $p_{c}$ and the arc length $u$. The mean-chord inequalities hold locally in the region I.

In the case $n=3$ the positivity of $S(n, u)$ is easily established, as the function $S(3, u)$ simplifies now to

$$
S(3, u)=2\left(2 \sin \frac{u}{2}\right)^{8}
$$

Since $T(2, u, p)<0$ holds for $p<p_{c}(u)$ the theorem is proven.

To visualize the result, in Figure 1 we plot the relation between the critical exponent $p_{c}$ given by (7) and the arc length $u$.

A comment is due on the closure of the curve $\Gamma$. In [2] the local validity of the inequality for $p=2$ was proved without this hypothesis. Here we used closure, but not to the full power of Proposition 1. We relied simply on the fact that the Fourier coefficients vanish for $|n| \leq 1$, which meant that the endpoints $\Gamma(0)$ and $\Gamma(2 \pi)$ meet within an error of $\mathcal{O}\left(\varepsilon^{2}\right)$, not $\mathcal{O}\left(\varepsilon^{3}\right)$.

Let us finally make one more remark, namely on a claim made in Thm. 5.4 
of [3]. It was stated there that for a particular class of deformations the circle remains a local maximizer for all $p$, namely for those which, in the complex notation, have the form $(1-\varepsilon) e^{i s}+\Theta(\varepsilon, s)$, with the assumption that for each $\varepsilon, \Theta(\varepsilon, s)$ is orthogonal to $e^{i s}$ and $\Theta(\varepsilon, s)$ is $\mathcal{C}^{2}$ smooth. In fact, the $\mathcal{C}^{2}$ assumption in the variable $\varepsilon$ cannot occur. To see that, notice that the condition $\int|\dot{\Gamma}(s)|^{2} \mathrm{~d} s=2 \pi$ together with orthogonality imply

$$
\int\left|\Theta_{s}(\varepsilon, s)\right|^{2} \mathrm{~d} s=4 \pi \varepsilon-2 \pi \varepsilon^{2}
$$

where $\Theta_{s}:=\partial \Theta / \partial s$. Since $\Theta$ is $\mathcal{C}^{2}$ by assumption, we may differentiate under the integral sign to get

$$
2 \operatorname{Re} \int \bar{\Theta}_{s}(\varepsilon, s) \frac{\partial \Theta_{s}(\varepsilon, s)}{\partial \varepsilon} \mathrm{d} s=4 \pi-4 \pi \varepsilon
$$

using the observation from [3] that $\Theta(0, s)=0$ we see that the left-hand side would tent to zero as $\varepsilon \rightarrow 0$ given the assumption that $\Theta$ is jointly $\mathcal{C}^{2}$, while the right-hand one has the nonzero limit $4 \pi$. To obtain smooth perturbations one should suppose, e.g., $\Gamma(\varepsilon, s)=\left(1-\varepsilon^{2}\right) e^{i s}+\Theta(\varepsilon, s)$, and this would necessitate an analysis to second order in $\varepsilon$, as has been done in this article.

\section{Acknowledgments}

The research was supported in part by the Czech Academy of Sciences and Ministry of Education, Youth and Sports within the projects A100480501 and LC06002.

\section{References}

[1] A. Abrams, J. Cantarella, J.G. Fu, M. Ghomi, R. Howard: Circles minimize most knot energies, Topology 42 (2003), 381-394.

[2] P.Exner: An isoperimetric problem for leaky loops and related meanchord inequalities, J. Math. Phys. 46 (2005), 062105

[3] P. Exner, E.M. Harrell, M. Loss: Inequalities for means of chords, with application to isoperimetric problems, Lett. Math. Phys. 75 (2006), 225233; addendum 77 (2006), 219 
[4] Jun O'Hara: Energy of Knots and Conformal Geometry, World Scientific, Singapore 2003.

[5] G. Lükö: On the mean lengths of the chords of a closed curve, Israel J. Math. 4 (1966), 23-32. 